\title{
Molecule-Type Phases and Hund's Rule in Vertically Coupled Quantum Dots
}

\author{
B. Partoens* and F. M. Peeters ${ }^{\dagger}$ \\ Departement Natuurkunde, Universiteit Antwerpen (UIA), B-2610 Antwerpen, Belgium
}

(Received 7 October 1999)

\begin{abstract}
We study the ground state of two vertically coupled quantum dots as a function of the interdot distance within the spin density functional theory. The tunneling between the dots is included. For small and large interdot distances the atomic phases are recovered. For intermediate distances new molecule-type phases are predicted which can be observed experimentally in the addition energies. The results are interpreted in terms of an effective single particle picture and we find that Hund's rule breaks down for 11 and 12 electrons. The results are summarized in a phase diagram in which spin and isospin blockade regions are also found.
\end{abstract}

PACS numbers: 73.20.Dx, 71.15.Mb, 73.23.Hk, 73.40.Gk

Quantum dots, or artificial atoms, have been the subject of intense theoretical and experimental research over the last few years [1]. Artificial atoms are small laboratory systems in which the effects of electron-electron interaction can be studied in a controlled way, because the dots' size, shape, and number of electrons can be varied. A more recent development is the study of vertically coupled dots [2-4] or artificial molecules, where also the distance between the dots can be changed.

Austing et al. [5] reported the fabrication of vertically coupled dots on which they performed single-electron tunneling experiments. Asano [6] studied the correlation effects in a double dot molecule, but considered the situation in which the Coulomb interaction energy is much smaller than the confinement energy and in which the lowest two bonding as well as antibonding shells are completely filled for all interdot distances. Both conditions are not fulfilled in typical GaAs quantum dots. Rontani et al. [7] calculated the ground state energies for different interdot distances using a general Hubbard approach and an exact diagonalization scheme up to six electrons. Mean-field methods are needed to simplify the complicated many-body problem when one wants to study more particles. In this Letter we therefore apply the spin density functional theory (SDFT) formalism which was successfully applied before to describe single quantum dots [8].
As shown by Weinmann et al. [9], the Coulomb oscillation peak corresponding to the transition from an $N$-electron quantum dot to an $(N+1)$-electron quantum dot is forbidden at low temperatures if the change in the total spin is $|\Delta S|>1 / 2$; this blocking effect is called the spin blockade. However, this spin blockade condition does not occur for circular quantum dots with parabolic confinement potential, but it can be realized in vertically coupled quantum dots.

The purpose of this Letter is to calculate the ground state of two vertically coupled quantum dots, with the interdot tunneling included. As we use the Kohn-Sham formalism it is natural to interpret the results in terms of an effective single particle picture supplemented by Hund's rule as in real atoms. The addition energies which are measured in single-electron tunneling experiments are calculated and we propose the suppression of some tunneling peaks due to spin and isospin blockades.

We consider two circularly symmetric dots, and assume a parabolic confinement potential $V(r)=\frac{1}{2} m \omega_{0}^{2} r^{2}$ of frequency $\omega_{0}$, with $m$ the effective electron mass. In the $z$ direction the quantum dots are created by two coupled quantum wells. The ground state energy and the density $\rho(\mathbf{r})$ are expressed in terms of a set of Kohn-Sham orbitals $\psi_{j l \sigma}(\mathbf{r})=\exp (-i l \theta) \varphi_{j l \sigma}(r) Z(z)$, which are the eigenstates of the $z$ component of the angular momentum $-l$, and satisfy the Kohn-Sham equation

$$
\begin{aligned}
{\left[-\frac{\hbar^{2}}{2 m}\left(\frac{\partial^{2}}{\partial r^{2}}+\frac{1}{r} \frac{\partial}{\partial r}-\frac{l^{2}}{r^{2}}+\frac{\partial^{2}}{\partial z^{2}}\right)+\frac{m \omega_{0}^{2} r^{2}}{2}+V(z)+V_{H}^{\text {intra }}(r)+V_{H}^{\text {inter }}(r)\right.} & \left.+\frac{\delta E_{x c}}{\delta \rho^{\sigma}(r)}\right] \varphi_{j l \sigma}(r) Z(z) \\
& =\epsilon_{j l \sigma} \varphi_{j l \sigma}(r) Z(z),
\end{aligned}
$$

with $\sigma$ being the $z$ component of the spin. The total density in the dots is $\rho(r)=\sum_{\sigma} \sum_{j, l}^{N_{\sigma}}\left|\varphi_{j l \sigma}(r)\right|^{2}$. Here we approximated the density in the $z$ direction by $\delta$ functions. Because both dots are identical the density in each dot is half this total density. The Kohn-Sham equation includes the intradot and interdot Hartree potentials:

$$
\begin{gathered}
V_{H}^{\text {intra }}(r)=\int d \mathbf{r}^{\prime} \frac{e^{2} \rho\left(r^{\prime}\right) / 2}{\varepsilon\left|\mathbf{r}-\mathbf{r}^{\prime}\right|}, \\
V_{H}^{\text {inter }}(r)=\int d \mathbf{r}^{\prime} \frac{e^{2} \rho\left(r^{\prime}\right) / 2}{\varepsilon\left|\mathbf{r}-\mathbf{r}^{\prime}+\mathbf{d}\right|},
\end{gathered}
$$

with $d=|\mathbf{d}|$ the interdot distance. $E_{\mathrm{xc}}$ is the exchange-correlation energy functional for which we 
use the local spin density approximation $E_{\mathrm{xc}}=$ $2 \pi \int \rho(r) \epsilon_{\mathrm{xc}}\left[\rho^{\uparrow}, \rho^{\downarrow}\right] r d r$, where the Tanatar-Ceperley [10] functional for $\epsilon_{\mathrm{xc}}\left(\rho^{\uparrow}, \rho^{\downarrow}\right)$ was used. The ground state energy of the double dot system is obtained from

$$
\begin{aligned}
E(N)= & \sum_{i \sigma} \epsilon_{i \sigma}-\frac{1}{2} \int \rho(r)\left(V_{H}^{\text {intra }}(r)+V_{H}^{\text {inter }}(r)\right) d \mathbf{r} \\
& -\sum_{\sigma} \int \rho^{\sigma}(r) \frac{\delta E_{\mathrm{xc}}\left[\rho^{\dagger} / 2, \rho^{\downarrow} / 2\right]}{\delta \rho^{\sigma}(r)} \\
& +2 E_{\mathrm{xc}}\left[\rho^{\uparrow} / 2, \rho^{\downarrow} / 2\right] .
\end{aligned}
$$

To solve the Kohn-Sham equation we expand the single particle wave functions $\varphi_{j l \sigma}$ in a Fock-Darwin basis.

For the material parameters we choose typical GaAs values $\left(m=0.067 m_{e}\right.$ and $\left.\varepsilon=12.4\right)$. This yields an effective Bohr radius of $a_{B}^{*}=9.79 \mathrm{~nm}$. The strength of the confinement is set to $\hbar \omega_{0}=5.78 \mathrm{~N}^{-1 / 4} \mathrm{meV}$. For different $N$, this choice keeps the average electron density in each dot approximately constant, in this case roughly corresponding to a (2D) Wigner-Seitz radius $r_{s}=1.75 a_{B}^{*}$, as in a typical vertical quantum dot [5].

The confinement in the $z$ direction consists of two coupled quantum wells. Because of the finite barrier between both dots the lowest level in the $z$ direction is split into a symmetric bonding and an antisymmetric antibonding level. Only these two lowest $z$ levels are included, and the contribution from excited states is neglected because the confinement in the $z$ direction is much stronger than in the plane. The motion in the $z$ direction may be assumed to be decoupled from the in-plane motion and therefore the $z$ direction in the Kohn-Sham equation can be solved separately. As an example we take the dimensions of the quantum wells corresponding to the experimental realization of Ref. [5]: $W=120 \AA$ for the width of both dots; $V_{0}=250 \mathrm{meV}$ for the height of the barrier between the dots, which results in the energy splitting $\Delta=22.86 \exp [-d(\AA) / 13.455] \mathrm{meV}$.

Only the interdot Hartree term is taken into account, and not the interdot exchange and correlation energy. Because the barrier between the dots is rather high, the overlap of wave functions localized in different dots is very small, which results in a negligible interdot exchange contribution. By comparing with the energy of a single quantum dot we estimated the error for not including the interdot correlation to be $<1 \%$ for $N>2$.

Figure 1 shows the calculated ground state energy as a function of $d$ and $\Delta$ for nine electrons in the system. The levels are labeled by the three quantum numbers $\left(S_{z}, M_{z}, I_{z}\right):$ spin $S_{z}$, angular momentum $M_{z}$, and an isospin quantum number $I_{z}$, which is the difference between the electrons in the bonding level and those in the antibonding level, divided by 2 . The isospin was first introduced by Palacios and Hawrylak [2] who showed that it is a good quantum number in the strong coupling limit, i.e., it is related to the symmetry operation of switching the individual electrons between the dots [2,4]. In the weak coupling limit the useful numbers are the number

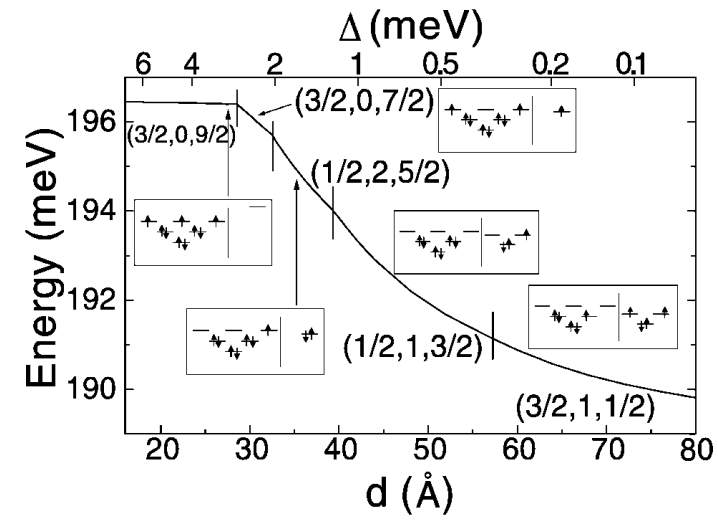

FIG. 1. The ground state energies as a function of $d$ and $\Delta$ for nine electrons. The levels are labeled with the quantum numbers $\left(S_{z}, M_{z}, I_{z}\right)$. The insets show the corresponding single particle picture.

of electrons in the left and the right dot which can be easily derived from the isospin quantum number. The ground state has spin $3 / 2$ and zero angular momentum for small interdot distances $(d<28.3 \AA)$, while for large interdot distances $(d>57.2 \AA)$ the angular momentum has changed to 1 . These phases can be understood from a single particle picture. The single particle energy levels $E_{n l}=\hbar \omega_{0}(2 n+|l|+1)$ (with $n$ the radial quantum number) are schematically shown in the insets of Fig. 1. Because of the splitting of the energy level in the $z$ direction, all these single particle levels are doubled and shifted by an energy $\Delta$. As shown in the insets these phases obey Hund's first rule: for a partially filled shell the total spin is maximized, which is a consequence of the exchange energy gain. For small interatomic distances the coupling between the dots is very strong, and they behave as a single dot, while for large interatomic distances the system behaves as two decoupled dots. However for intermediate distances three new molecule-type phases are found.

The number of new molecule-type phases increases with the number of electrons. For four, five, and six electrons one intermediate molecule-type phase is found, corresponding to the results of Ref. [7]. For seven and eight electrons two intermediate molecule-type phases were found. The existence of all these phases can be understood in terms of the single particle picture supplemented with Hund's rule, including the energy shift $\Delta$ and the electron-electron interaction, which lifts the degeneracy of the single particle levels in a shell, to explain the angular momentum of the state with maximum spin. For example, the highest filled single particle level for seven electrons for the case of very small interdot distance is the $(n=0, l=2)$ level. The level $(n=1, l=0)$ has a higher energy caused by electron-electron interaction due to the large overlap with the filled level with the same angular momentum $(n=0, l=0)$. This lifting of the degeneracy creates an extra molecule-type phase for the case of $N=9$, namely, when the interdot distance decreases the transition from the atomic phase to the 
first molecule-type phase occurs when the electron in the bonding $(n=1, l=0)$ level hops to the lowest antibonding level. A naive use of Hund's rule would predict that at the first transition two electrons would hop together to the lowest antibonding level. But the degeneracy of the single particle levels in a shell is lifted due to electron-electron interaction, and the smaller the angular momentum the larger the energy increase, and as a consequence Hund's rule still works.

Although the interpretation of the results in terms of a single particle picture is natural in the used Kohn-Sham scheme, it is important to stress that the real levels are linear combinations of these single particle states.

In Fig. 2 a phase diagram of all transitions up to 13 electrons is presented. Notice that for $N=10$ we find two intermediate molecule-type phases, which is one less than for the $N=9$ case. The reason is that for $d \approx 40 \AA$ two electrons hop together from bonding to antibonding levels. In contrast, for $N=11$ there are six transitions, while Hund's rule predicts the existence of only five transitions, namely, each time when an electron in a bonding level hops to an antibonding level. This extra phase can be understood from the schematic picture of Fig. 3. For $I_{z}=7 / 2$ Hund's rule predicts $S_{z}=3 / 2$ and $M_{z}=0$ as

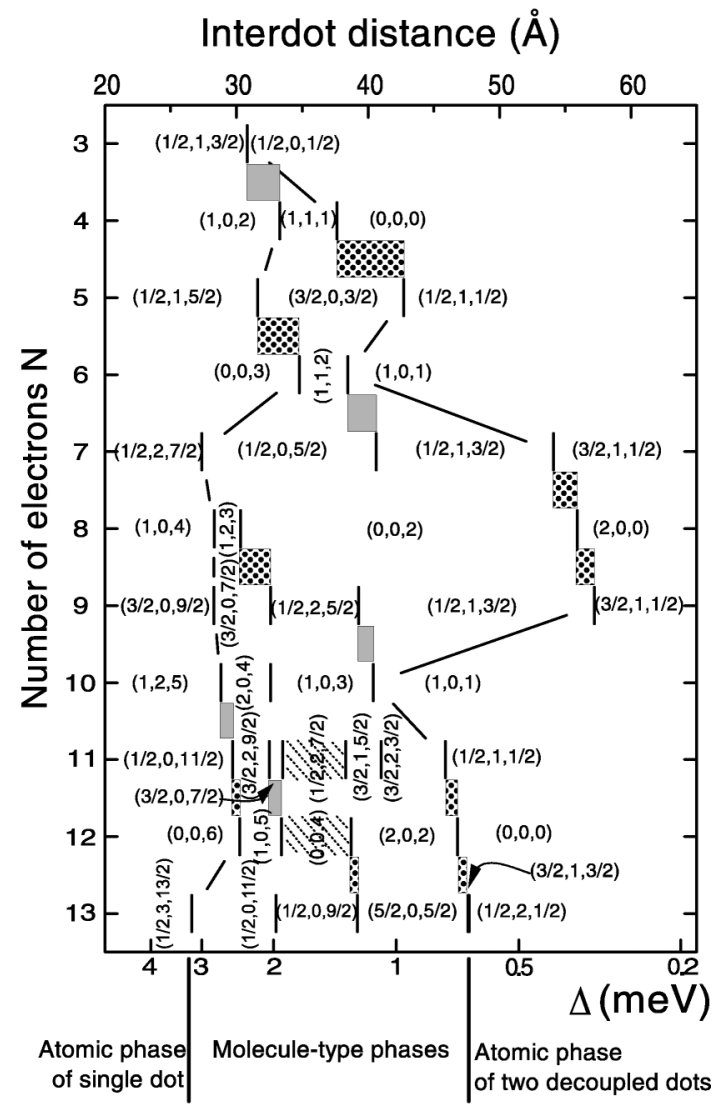

FIG. 2. Phase diagram for up to 13 electrons, labeled by the quantum numbers $\left(S_{z}, M_{z}, I_{z}\right)$. The region between the lines marks the region of new molecule-type phases. The areas with dashed lines are the phases for which Hund's rule breaks down. The dotted (shaded) areas mark the spin (isospin) blockade regions. ground state [Fig. 3(a)]. However at some interdot distance it is energetically favorable that the electron in the highest bonding $l=0$ level hops to a bonding $l=2$ level, resulting in $S_{z}=1 / 2$ and $M_{z}=2$ [Fig. 3(b)]. The reason is that this bonding $l=0$ and the two filled antibonding $l=0$ levels have a large overlap, which increases the level splitting. The exchange energy gain in the case of three parallel spins is not large enough to overcome this splitting, which results in this extra phase. Thus Hund's rule breaks down in two vertically coupled dots starting from 11 electrons, while for single dots it was found [8] that Hund's rule breaks down starting from 24 electrons.

In the $N=12$ case Hund's rule also breaks down, but now as the absence of a molecule-type phase near $d=35 \AA$ where $I_{z}=4$. Hund's rule predicts the phase $(1,2,4)$, but we found that $(0,0,4)$ has a lower energy. The physical explanation is the same as for $N=11$.

For 13 electrons all molecule-type phases can be predicted from the single particle picture with Hund's rule together with tunneling. The $l=0$ electron in the second bonding shell in the molecule-type phase $(5 / 2,0,5 / 2)$ never hops to a higher angular momentum level in that shell. The exchange energy gain is now large enough (five aligned spins) to overcome the level splitting and Hund's rule does not break down.

Signatures of the above molecule-type phases can be measured experimentally through the addition energies. The addition energy is the energy needed to place one more electron in the coupled dots, i.e., we calculated $\quad \Delta \mu(N)=\mu(N+1)-\mu(N)=E(N+1)-$ $2 E(N)+E(N-1)$. Figure 4(a) shows the addition energies for the cases of small, intermediate, and large interdot distances, as a function of the number of electrons. Note that for a small interdot distance (i.e., $d=15 \AA$ ) the peaks at 2, 4, 6, 9, 12, . reflect the shell structure of a 2D harmonic oscillator. For large interdot distances the dots are decoupled and the magic numbers are doubled, i.e., the peaks are observed at $N=4,8,12, \ldots$ These magic numbers disappear for intermediate interdot distance.
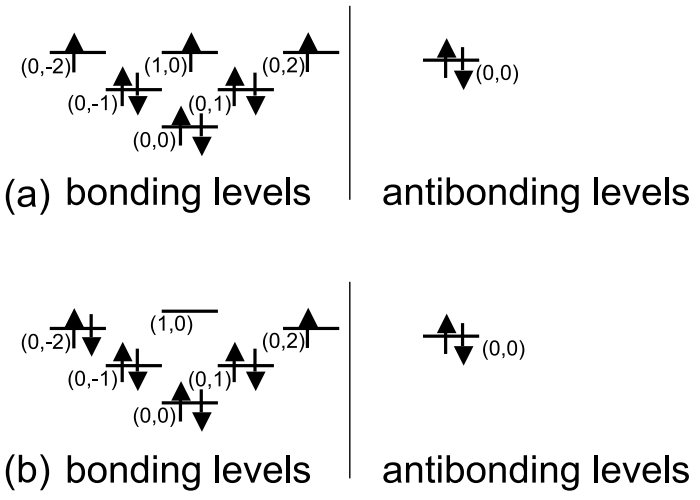

FIG. 3. Single particle pictures for 11 electrons in the case of $I_{z}=7 / 2$. (a) is the molecule-type phase as predicted by Hund's rule, and (b) is the extra predicted molecule-type phase. Each level is labeled with the single particle quantum numbers $(n, l)$. 

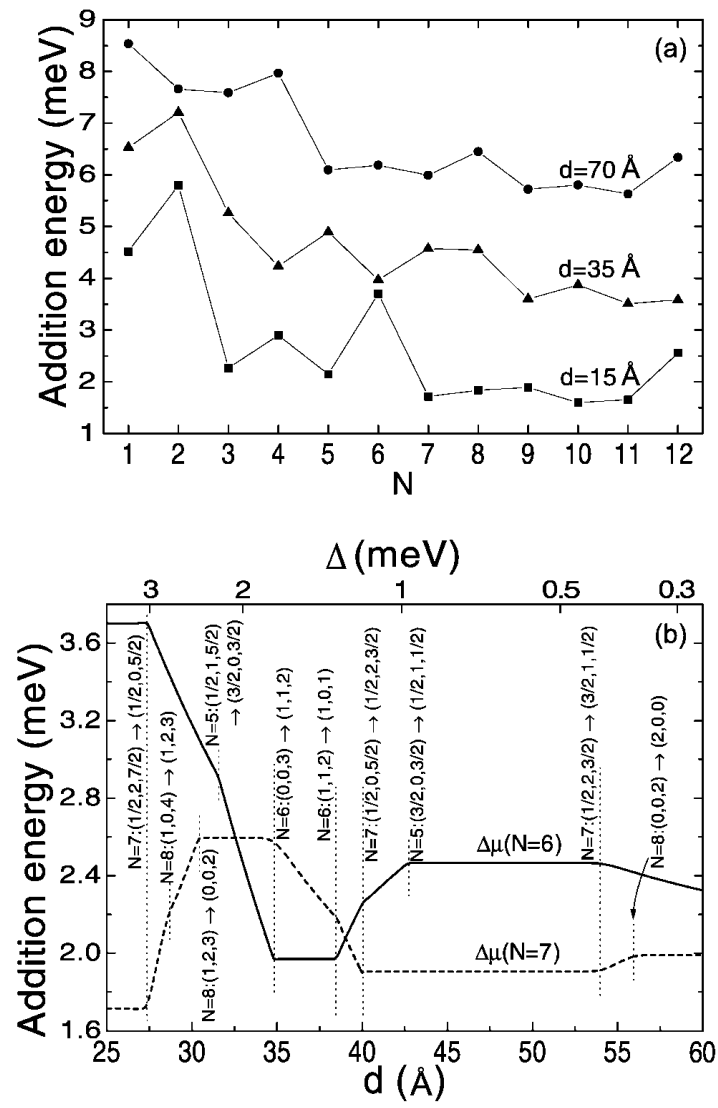

FIG. 4. The addition energies as a function of the number of electrons for three interdot distances. The curves for $d=$ 35 and $d=70 \AA$ are shifted by 2 and $4 \mathrm{meV}$, respectively. (b) The addition energies for six and seven electrons as a function of $d$ and $\Delta$. The labels at the kinks of the curves indicate which transition occurs between which two phases and for how many electrons in the double dot.

How the addition energies change continuously from these two atomic limit cases is shown in Fig. 4(b) for six and seven electrons in the system. It shows the addition energy as a function of the interdot distance. One can see how the magic number 6 disappears, while the addition energy for $N=7$ increases. This modification of the shell structure is due to the appearance of new moleculetype phases and the kinks in these curves correspond to transitions between these phases. For example, the first kink occurs at $d \approx 27 \AA$ in both curves $\Delta \mu(6)$ and $\Delta \mu(7)$ and it corresponds to the transition between the phases $(1 / 2,2,7 / 2) \rightarrow(1 / 2,0,5 / 2)$ for $N=7$ electrons. In this way all kinks are labeled in Fig. 4(b) with the ground state phases between which the transition occurs, i.e., the quantum numbers and the number of electrons are given. The flat regions in the addition energy occur when the level splitting energies $\Delta$ cancel in the calculation of $\Delta \mu(N)$.

The spin blockade condition is $|\Delta S|>1 / 2$. But also in the case $|\Delta S|=1 / 2$ the Coulomb oscillation peak can be suppressed if some of these tunneling channels are blocked which occurs when $\left|\Delta S_{z}\right|>1 / 2$ [11]. This condition is much easier satisfied in two coupled quantum dots than in a single parabolic dot. The spin blockade regions are indicated in the phase diagram of Fig. 2 by the dotted areas. The same is of course true for the isospin quantum number for which an isospin blockade may be observed (shaded areas in Fig. 2). In Ref. [4] the existence of an isospin blockade region was predicted from an exact diagonalization calculation for the transition from $N=3$ to $N=4$. This result is precisely reproduced in our SDFT calculations, which justifies our approach.

In conclusion, we applied spin density functional theory to describe two vertically coupled quantum dots, including tunneling. For small and large interdot distances we recover the atomic phases, while for intermediate distances new molecule-type phases are found. The occurrence of the atomic and molecule-type phases can be understood from Hund's rule. However, this single particle picture cannot predict the interdot distances at which the transitions occur. We also found that Hund's rule breaks down starting from 11 electrons in the coupled dots. The addition energies which can be measured experimentally in single-electron tunneling experiments were calculated and the new molecule-type phases should be observable in these addition spectra. It is also shown that spin and isospin blockade regions are much more easily realizable in coupled dots than in single dots.

This work is supported by the Flemish Science Foundation (FWO-Vl) and IUAP-IV.

*Electronic address: bpartoen@uia.ua.ac.be

†Electronic address: peeters@uia.ua.ac.be

[1] L. Jacak, P. Hawrylak, and A. Wójs, Quantum Dots (Springer-Verlag, Berlin/Heidelberg, 1998).

[2] J. J. Palacios and P. Hawrylak, Phys. Rev. B 51, 1769 (1995).

[3] H. Imamura et al., Phys. Rev. B 53, 12613 (1996); 59, 5817 (1999); S. C. Benjamin and N. F. Johnson, Phys. Rev. B 51, 14733 (1995); J.H. Oh et al., Phys. Rev. B 53, 13264 (1996); W.-Y. Ruan and H.-F. Cheung, Eur. Phys. J. B 3, 407 (1998); B. Partoens et al., Phys. Rev. Lett. 79, 3990 (1997).

[4] Y. Tokura, D. G. Austing, and S. Tarucha, J. Phys. Condens. Matter 11, 6023 (1999).

[5] D. G. Austing et al., Physica (Amsterdam) 249B-251B, 206 (1998).

[6] Y. Asano, Phys. Rev. B 58, 1414 (1998).

[7] M. Rontani et al., Solid State Commun. 112, 151 (1999).

[8] M. Koskinen et al., Phys. Rev. Lett. 79, 1389 (1997); K. Hirose and N. S. Wingreen, Phys. Rev. B 59, 4604 (1999); M. Ferconi and G. Vignale, Phys. Rev. B 50, 14722 (1994); O. Steffens and M. Suhrke, Phys. Rev. Lett. 82, 3891 (1999); S. Nagaraja et al., Phys. Rev. B 60, 8759 (1999); I.-H. Lee et al., Phys. Rev. B 57, 9035 (1997); M. Stopa, Phys. Rev. B 54, 13767 (1996).

[9] D. Weinmann et al., Phys. Rev. Lett. 74, 984 (1995).

[10] B. Tanatar and D. M. Ceperley, Phys. Rev. B 39, 5005 (1989).

[11] B. Jouault et al., cond-mat/9911224. 